\title{
NETI-mediated RhoA activation facilitates lysophosphatidic acid-induced cell migration and invasion in gastric cancer
}

\author{
D Murray', G Horgan², P MacMathuna ${ }^{2}$ and P Doran*,1 \\ 'UCD Clinical Research Centre, UCD School of Medicine and Medical Sciences, Mater Misericordiae University Hospital, 49-5 I Eccles Street, Dublin 7, \\ Ireland; ${ }^{2}$ Gastrointestinal Unit, Mater Misericordiae University Hospital, Dublin 7, Ireland
}

The most lethal aspects of gastric adenocarcinoma (GA) are its invasive and metastatic properties. This aggressive phenotype remains poorly understood. We have recently identified neuroepithelial cell transforming gene I (NETI), a guanine exchange factor (GEF), as a novel GA-associated gene. Neuroepithelial cell transforming gene I expression is enhanced in GA and it is of functional importance in cell invasion. In this study, we demonstrate the activity of NETI in driving cytoskeletal rearrangement, a key pathological mechanism in gastric tumour cell migration and invasion. Neuroepithelial cell transforming gene I expression was increased I0-fold in response to treatment with lysophosphatidic acid (LPA), resulting in an increase in active levels of RhoA and a 2-fold increase in cell invasion. Lysophosphatidic acid-induced cell invasion and migration were significantly inhibited using either NETI siRNA or a RhoA inhibitor (C3 exoenzyme), thus indicating the activity of both NETI and RhoA in gastric cancer progression. Furthermore, LPA-induced invasion and migration were also significantly reduced in the presence of cytochalasin D, an inhibitor of cytoskeletal rearrangements. Neuroepithelial cell transforming gene I knockdown resulted in AGS cell rounding and a loss of actin filament organisation, demonstrating the function of NETI in actin organisation. These data highlight the importance of NETI as a driver of tumour cell invasion, an activity mediated by RhoA activation and cytoskeletal reorganisation.

British Journal of Cancer (2008) 99, I 322-1329. doi:I0.1038/sj.bjc.6604688 www.bjcancer.com

Published online 30 September 2008

(c) 2008 Cancer Research UK

Keywords: NETI; LPA; gastric cancer; invasion; migration; RhoA

Gastric adenocarcinoma (GA) is the second most significant cause of cancer-related deaths worldwide (Hohenberger and Gretschel, 2003). Tumour metastasis is the most lethal aspect of this disease, whereby tumour cells spread to local or often distant lymph nodes. Furthermore, lymph node involvement in GA greatly affects disease prognosis and is associated with poor outcome (de Manzoni et al, 2001; Hohenberger and Gretschel, 2003). The invasive and metastatic abilities of these tumours and more specifically the molecular mechanisms underpinning this aggressive phenotype represent ideal targets for therapeutic intervention.

Tumour metastasis is a complex multistep process, involving detachment of cells from the primary tumour, local invasion through surrounding tissue and basement membrane, followed by intravasation into the circulatory system and ultimately extravasation and growth at distant organs. At the molecular level, tumour invasion and metastasis is dependent on the complex interplay between key biomolecules, including components of the extracellular matrix, cell adhesion molecules and proteolytic enzymes, all of which contribute to the pathological spread of tumour cells into neighbouring tissues and extracellular spaces (Poste and Fidler, 1980). Regulation of the tumour cell cytoskeleton to facilitate invasion is also an important phenotypic change in metastatic tumour cells.

The motility of tumour cells is dependent on rearrangements of the actin cytoskeleton for generating both protrusions and retraction to generate a motility cycle resulting in net translocation

*Correspondence: Dr P Doran; E-mail: peter.doran@ucd.ie

Received 16 April 2008; revised I September 2008; accepted I September 2008; published online 30 September 2008
(Wang et al, 2005). Rho GTPases are an important family of proteins that regulate the actin cytoskeleton. Modulation of Rho activity promotes the metastasis of tumour cells by disrupting epithelial-sheet organisation, increasing cell motility and promoting the degradation of the extracellular matrix (Sander et al, 1998; Sahai and Marshall, 2002).

We have recently identified enhanced NET1 expression in GA in comparison with adjacent normal tissue and we have furthermore shown NET1 to play a role in tumour cell invasion (Leyden et al, 2006). Neuroepithelial cell transforming gene 1 is a member of the guanine nucleotide exchange factor (GEF) family, a group of proteins that are known to activate and thereby regulate Rho family members (Symons and Rusk, 2003; Rossman et al, 2005). Although the roles of other GEFs in other cancers have been established, the role of NET1 has not yet been elucidated. Other GEFs with established roles in various malignancies include; ASEF, which has been shown to promote the migration of colorectal cancer cells (Kawasaki et al, 2003; Nathke, 2006); Bcr, which by chromosome translocation and the formation of the Philadelphia chromosome and the BcrAbl fusion protein is essential for oncogenesis in human leukaemias (Advani and Pendergast, 2002). Another GEF, GEFH1, has been shown recently to be transcriptionally responsive to mutant p53 that resulted in increased tumour cell proliferation in a model of osteosarcoma (Mizuarai et al, 2006). Although NET1 was originally identified as an oncogene in neuroepithelial cells, its functional importance in other malignancies has not yet been established (Chan et al, 1996). Rho GTPases comprise a main branch of the Ras superfamily of small $(\sim 21 \mathrm{kDa})$ GTPases and function as bimolecular switches, changing conformational states in response to either GDP or GTP 
binding. Rho proteins are inactive when bound to GDP and active when GTP-bound, actively transducing signals by interaction with downstream effector proteins (Bishop and Hall, 2000). Binding to GTP and therefore activation is promoted by RhoGEFs and GTP hydrolysis, and therefore inactivation is catalysed by Rho-GTPase-activating proteins (Rho-GAPs). Interestingly, NET1 mRNA expression has been shown to be upregulated in response to Helicobacter pylori infection, an established event in gastric carcinogenesis (Chiou et al, 2001). In this study we aimed to further characterise the role of NET1 in GA. The effect of NET1 knockdown on RhoA activation was assessed. Furthermore, the effects of lysophosphatidic acid (LPA), a known activator of RhoA, on NET1 expression and cell motility were also investigated. The role of NET1 in LPA-induced RhoA activation and subsequent cell motility was assessed. Likewise, the role of cytoskeletal rearrangements in LPA-induced invasion and migration were investigated and the effect of NET1 knockdown on the actin cytoskeleton was also assessed. We aimed to characterise the function of NET1 in the gastric tumour setting by defining the mechanism underpinning its effect in promoting tumour cell invasion.

\section{MATERIALS AND METHODS}

\section{Cell culture and cell treatments}

AGS gastric cancer cell lines were cultured in Hams F12 medium supplemented with $10 \%$ fetal bovine serum and grown under standard conditions as previously described (Leyden et al, 2006). Throughout this study, cells were treated with the following: $20 \mu \mathrm{M}$ LPA for $4 \mathrm{~h}$; $4 \mu \mathrm{g} \mathrm{ml}^{-1} \mathrm{C} 3$ exoenzyme for $4 \mathrm{~h}$ or $5 \mu \mathrm{m}$ cytochalasin D (CyD).

\section{Gene silencing by RNA interference}

Neuroepithelial cell transforming gene 1 mRNA was silenced as previously described (Leyden et al, 2006). Briefly, siRNA duplexes were designed and synthesised for transiently silencing NET1, and a chemically synthesised non-silencing siRNA duplex that had no known homology with any mammalian gene was used to control nonspecific silencing events (Qiagen Inc., Valencia, CA, USA). The sequences were NET1 sense, 5'-GGAGGAUGCUAUAUUGAUA-3'; NET1 antisense, $5^{\prime}$-UAUCAAUAUAGCAUCCUCC- $3^{\prime}$ and non-targeting sense, $5^{\prime}$-UU CUCCGAACGUGUCACGU-3'; antisense, 5'-ACGUGACACGUUCG GAGAA-3'. RNA interference was performed in six-well format by seeding $3 \times 10^{5}$ cells per well. The effects of various doses of siRNA on mRNA production were investigated $(0,17,34$ and $75 \mathrm{nM}$ ) in a ratio of $1 \mu \mathrm{g}$ siRNA to $6 \mu \mathrm{l}$ to RNAifect (Qiagen) for $48 \mathrm{~h}$. RNA and protein were extracted and analysed as described below. All RNA interference (RNAi) experiments were repeated in triplicate. Separate RNAi treatments were performed to investigate any cytotoxic effect, briefly, following RNAi treatment, viability was assessed by trypsining and counting cells using Trypan blue staining and a haemocytomoeter. To decipher any off target effects, a second NET1 siRNA duplex was used with the following sequence: sense, $5^{\prime}$-GGUGUGGAUUGAUUGGAAA-3'; antisense, $5^{\prime}$-UUUCCAAUCAAUCCACACC- $3^{\prime}$.

\section{Flow cytometry}

The effect of RNAi on cell viability was assessed using flow cytometry by staining with propidium iodide and Annexin V FITC. Briefly, following knockdown, cells were trypsinised and washed twice in ice-cold phosphate-buffered saline (PBS). A total of $1 \times 10^{5}$ cells were resuspended in $100 \mu \mathrm{l}$ binding buffer $(10 \mathrm{~mm}$ HEPES/NaOH pH7.4, $140 \mathrm{~mm} \mathrm{NaCl}, 2.5 \mathrm{~mm} \mathrm{CaCl}_{2}$ ) to which was added $5 \mu \mathrm{l} 2 \mathrm{mg} \mathrm{ml}^{-1}$ Annexin V FITC and $10 \mu \mathrm{l} 50 \mu \mathrm{g} \mathrm{ml}^{-1}$ propidium iodide. Following $15 \mathrm{~min}$ incubation in the dark, flow cytometry was performed using a Cyan ADP analyzer (Dako, Dublin, Ireland) using 515/545 nm filter set for FITC detection and
$620 / 640 \mathrm{~nm}$ set for propidium iodide. All analysis was repeated in triplicate.

\section{RNA extraction and PCR}

TRIzol $^{\text {TM }}$ (Sigma-Aldrich, Dublin, Ireland) was used to extract RNA as previously described (Leyden et al, 2006). Following reverse transcription, as previously described (Leyden et al, 2006) real-time PCR was performed using a QuantiTect ${ }^{\mathrm{TM}}$ SYBR Green PCR Kit (Qiagen) following the manufacturers' instructions. Briefly, $2 \mu \mathrm{l}$ of cDNA template was mixed with $12.5 \mu \mathrm{l}$ SYBR Green master mix containing Taq and dNTPs (Qiagen), $8.5 \mu$ DNase-free water and $1 \mu \mathrm{l}$ each of forward and reverse primers. The sequences of primers used for PCR were $\beta$-actin forward: $5^{\prime}$-GTCACCTT CACCGTTCCAG-3', reverse: 5'-CTCTTCCAGCCTTCCTTCCT-3', NET1 forward: $5^{\prime}$-CTGTTCACCTCGGGACATTT- $3^{\prime}$, reverse: $5^{\prime}$-TG GAGCTGTCAGACGTTTTG- $3^{\prime}$. The reaction was carried out using a Rotor Gene ${ }^{\mathrm{TM}} 3000$ multiplex system. All measurements were independently repeated three times. $\beta$-Actin mRNA expression levels were used to normalise and compare expression values for the genes of interest. The PCR products were separated on $1 \%$ agarose gels and visualised under UV light.

\section{Guanosine tri-phosphate-RhoA pulldown and immunoblot analysis}

Guanosine tri-phosphate-bound RhoA was detected using a Rhotekin Rho-binding domain (RBD) 'pull down' assay (Upstate Inc., Lake Placid, NY, USA). Following NET1 knockdown, media was removed from cells before they were washed twice in ice-cold PBS and lysed in ice-cold $\mathrm{Mg}^{2+}$ lysis buffer (MLB) (Upstate Inc.). Cell lysates were clarified by centrifugation at $14000 \mathrm{~g}$ for $5 \mathrm{~min}$ at $4{ }^{\circ} \mathrm{C}$ and incubated with agarose-bound Rhotekin RBD beads (Upstate Inc.) at $4{ }^{\circ} \mathrm{C}$ for $45 \mathrm{~min}$. The beads were pelleted by centrifugation and washed three times in MLB before electrophoresis on a $13 \%$ SDSPAGE gel. Bound RhoA was detected by immunoblot using an antiRho monoclonal antibody (Upstate Inc.). Likewise active RhoB and RhoC levels were assessed using monoclonal antibodies ab53743 and ab54837, respectively (Abcam plc, Cambridge, UK). The amount of RBD-bound Rho was normalised to the total amount of Rho in total cell lysates for the comparison of Rho activity (level of GTP-bound Rho) in different samples. Neuroepithelial cell transforming gene 1 protein was detected using a monoclonal (H70) antibody (Santa Cruz Inc., Santa Cruz, CA, USA) at a 1:500 dilution. $\beta$-Actin protein levels were detected for use as a loading control using a monoclonal antibody (Merck Biosciences, Nottingham, UK). Densitometric analysis was performed using Image $1.39 \mathrm{u}$ software (NIH, USA). Using this software the density of the active Rho band was expressed as a ratio to the density of the total Rho band.

\section{Wound-healing migration assay}

To investigate the migratory capacity of cells, cells were grown to $100 \%$ confluence in six-well plates in the presence of NET1-specific or scrambled siRNA. The monolayer of cells were wounded by performing a scratch with a sterile $10 \mu \mathrm{l}$ micropipette tip. Cells were washed with sterile PBS, and fresh siRNA-containing media was then added. Cells were photographed under $\times 10$ objective lens at $24 \mathrm{~h}$. Carnoy software (Biovolution) was used to measure the pixel width of the scratches.

\section{In vitro trans-well migration and invasion}

Briefly, Biocoat Matrigel 8-micron invasion chambers (BD Biosciences, Bedford, MA, USA) were used to investigate the effect of cell treatments on the in vitro invasiveness of the AGS gastric cell line over $24 \mathrm{~h}$ as previously described (Leyden et al, 2006). Briefly, $5 \times 10^{5}$ cells were added to Biocoat Matrigel (BD 
Biosciences) 24-well invasion chambers and allowed to invade for $24 \mathrm{~h}$ under standard culture conditions. A chemo-gradient was established by seeding cells in $1 \%$ serum and adding $20 \%$ serum to the outer chamber. Invasive cells were fixed and stained using methanol and haematoxilyn as previously described (Leyden et al, 2006). Invasive cells were counted in five $\times 10$ magnification fields. Results were expressed as average cell count per field. Similarly, cell migration was monitored using 8-micron inserts without the inclusion of Matrigel.

\section{Fluorescent staining of cytoskeleton components}

Imunofluorescent staining for F-actin assembly was carried out using rhodamine phalloidin (FAK100 kit from Chemicon International Inc., Temecula, CA, USA). Cells were grown and treated as detailed above. All growth medium was removed and cells were washed three times for $5 \mathrm{~min}$ with $0.05 \%(\mathrm{v} / \mathrm{v})$ Tween-20 in PBS at room temperature. Cells were fixed for $15 \mathrm{~min}$ in $4 \%(\mathrm{w} / \mathrm{v}) \mathrm{p}$ formaldehyde in PBS at room temperature. Cells were washed as above and then permeabilised for 5 min with $0.1 \%$ Triton X-100 (v/ v) in PBS at room temperature. Cells were washed and then blocked for 30 min with $1 \%$ (w/v) BSA in PBS at room temperature. Following another wash step, labelling was achieved by incubating for $1 \mathrm{~h}$ with $0.12 \mu \mathrm{g} \mathrm{ml}^{-1}$ tetramethyl rhodamine isothiocyanate (TRITC)-conjugated phaloidin in PBS at room temperature in the dark. Cells were washed as above, and slides were mounted using an anitfade mounting solution (Cat no. 5013 from Chemicon International Inc.). Fluorescent images were visualised using a Zeiss Axioshop 40FL (Carl Zeiss Inc., Oberkochen, Germany).

\section{Statistical analysis}

Data are expressed as mean \pm s.d. from triplicate experiments and were analysed with Microsoft Excel using a student's $t$-test with significance defined as $P<0.05$.

\section{RESULTS}

The effect of NET1 on RhoA activation, gastric cancer cell migration and invasion

Loss-of-function experiments were carried out to determine the role of NET1 activity in gastric cancer. An RNAi approach using an siRNA duplex specifically targeting NET1 mRNA was used to investigate the effect of NET1 knockdown on RhoA activation and AGS gastric cancer cell migration and invasion. Neuroepithelial cell transforming gene 1 mRNA production was dose-responsive to siRNA, with $75 \mathrm{~nm}$ siRNA causing $54 \%$ reduction in mRNA expression $(P<0.05)$, whereas non-targeting siRNA caused no significant change in mRNA expression. (Figure 1A). This optimal dose was used in further experiments. The PCR products were also analysed by agarose gel electrophoresis (Figure 1B), where cells treated with 34 and $75 \mathrm{~nm}$ NET1 siRNA displayed decreased levels of NET1 mRNA in comparison with cells treated with the same concentration of non-targeting siRNA. Western immunoblot confirmed reduced NET1 protein levels (Figure 1C, first panel). To ensure RNAi treatments were non-cytotoxic, the viability of cells undergoing all treatments were compared using Trypan blue staining and cell counting. No significant differences were observed between groups (data not shown). Furthermore, there was no significant difference in cell viability numbers between $75 \mathrm{nM}$ control and NET1 siRNA groups as measured by flow cytometry using Annexin V-FITC and propidium iodide staining (Supplementary Figure 1). Reduced NET1 mRNA resulted in an attenuation of RhoA activation (Figure 1C). The level of activated RhoA protein was dramatically reduced in response to NET1 knockdown. Neuroepithelial cell transforming gene 1 knockdown also caused a less dramatic decrease in total RhoA levels. By expressing active RhoA as a ratio against
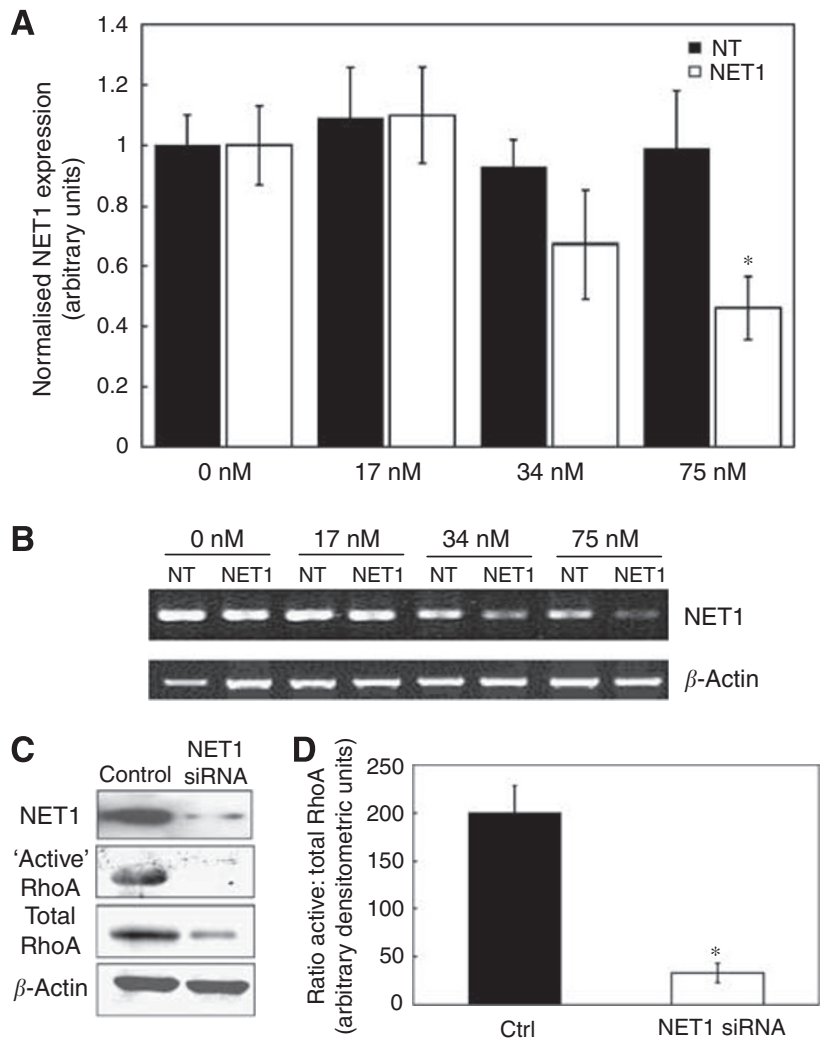

Figure I Decreased NETI results in a reduction in the level of active RhoA. (A) Dose-response of NETI mRNA expression to increasing levels of siRNA. Real-time PCR of NETI mRNA expression in AGS cells following treatment with 0, 17, 34 or $75 \mathrm{nM}$ non-target (NT) or NETIspecific siRNA in six-well format. (B) Agarose gel analysis of NETI and $\beta$-actin PCR products in samples treated with or without increasing doses of NETI or non-target (NT) siRNA. (C) Western blot analysis of control and RNAi-treated AGS cells. First panel: western immunoblot analysis of NETI protein. Second and third panel: 'active' and total RhoA, respectively Fourth panel: $\beta$-actin (loading control). (D) Ratio of active RhoA: tota RhoA protein in control and NETI siRNA samples, as determined using densitometric analysis. ( $P<0.05)$. Error bars represent standard deviation of triplicate experiments.

total RhoA using densitometric analysis, an $84 \%$ reduction $(P<0.05)$ in this ratio was observed in cells treated with NET1 siRNA (Figure 1D). A second NET1 targeting siRNA duplex combination also resulted in decreased levels of active RhoA (Supplementary Figure 2). Knockdown of NET1 did not result in a change in the levels of active RhoB or RhoC (Supplementary Figure 3).

The functional importance of NET1 in gastric cancer progression was displayed by a suppressive effect of NET1 knockdown on AGS cell migration (Figure 2A). Using a wound-healing assay, AGS cells in which NET1 knockdown was achieved were observed to be less migratory than control cells (Figure 2A). There was no significant wound healing in NET1 knockdown cells. After $24 \mathrm{~h}$, control cells migrated and thereby reduced the wound width by $42 \%(P<0.005)$, where NET1 knockdown cells migrated and reduced the wound width $9 \%$ (Figure $2 \mathrm{~B}$ ). Using the trans-well system, $81 \%$ decreased in vitro migration and $94 \%$ decreased invasion was observed in AGS cells in which NET1 knockdown had been achieved $(P<0.05)$ (Figure $2 \mathrm{C}$ and $\mathrm{D})$.

\section{The role of RhoA activation in NET1-mediated gastric cancer cell migration and invasion}

Having shown a relationship between NET1 expression and RhoA activity, the effect of LPA, a known activator of RhoA, on NET1 
A
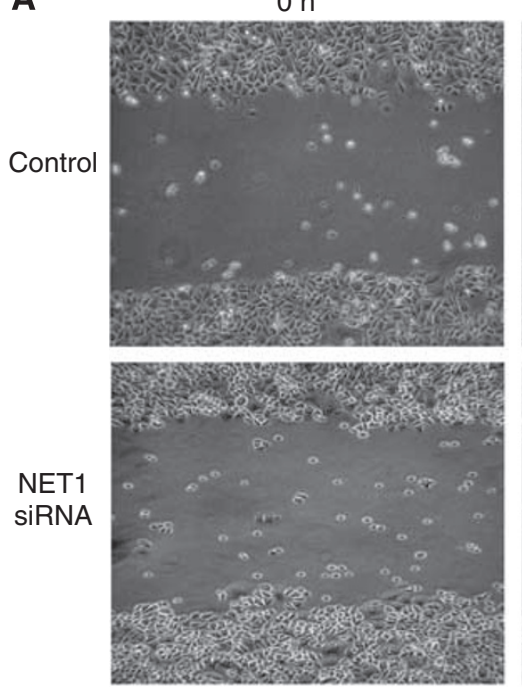

$24 \mathrm{~h}$
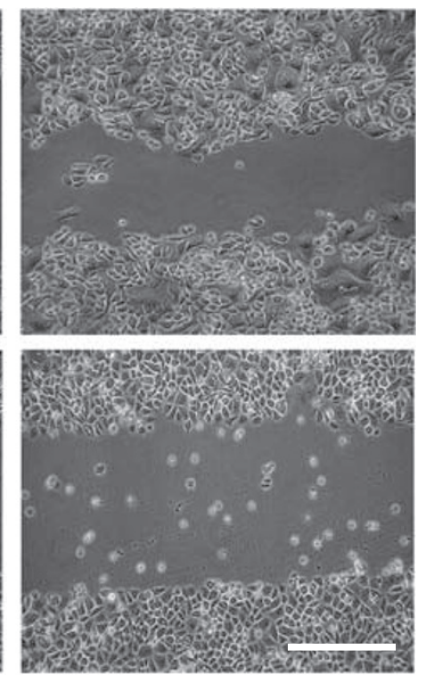

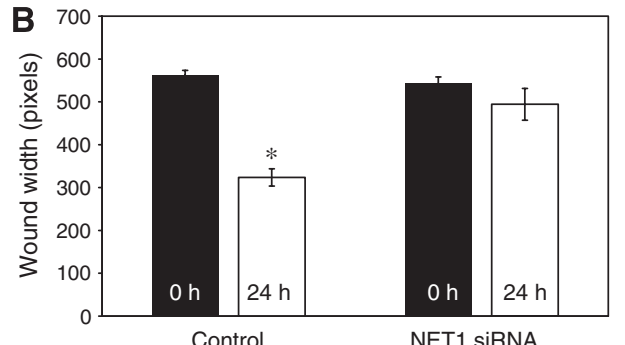

Control

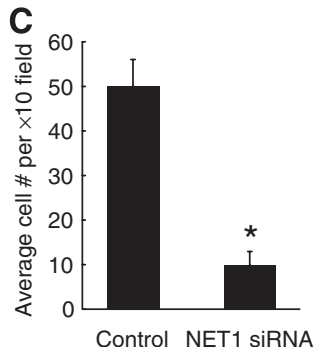

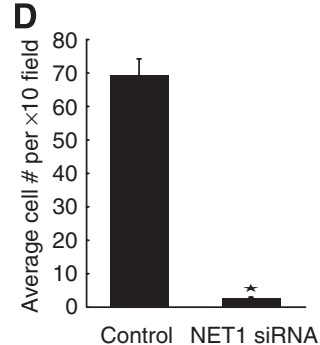

Figure 2 Knockdown of Netl mRNA expression results in decreased AGS cell migration and invasion. (A) The effect of RNAi-mediated NETI knockdown on AGS cell migration using a wound-healing assay in comparison with control AGS cells. Cells were treated with targeting or non-targeting siRNA for $48 \mathrm{~h}$ before the wound-healing assay. Cell migration was measured $24 \mathrm{~h}$ after the 'wound'. Bar: $200 \mu \mathrm{m}$. (B) Graphical representation of the effect of NETI siRNA on wound healing. (C) The in vitro migratory capacity of control and NETI knockdown cells using trans-well migration chambers. Cells were treated with targeting or non-targeting siRNA for $48 \mathrm{~h}$ before the migration assay, equal numbers of cells $\left(5 \times 10^{4}\right)$ were added to the chambers to determine the effect of knockdown on migration over $24 \mathrm{~h}$. (D) The in vitro invasive ability of control and NETI knockdown cells using trans-well Matrigel invasion chambers. Cells were treated with targeting or non-targetting siRNA for $48 \mathrm{~h}$ before the migration assay, equal numbers of cells $\left(5 \times 10^{4}\right)$ were added to the trans-well chambers to determine the effect of knockdown on invasion over $24 \mathrm{~h}$. (*P<0.05). Error bars represent standard deviation of triplicate experiments.

expression was investigated. Lysophosphatidic acid was shown to increase NET1 mRNA expression in a dose-dependent manner in AGS gastric cancer cells (Figure 3A). Likewise, treatment with LPA resulted in a dose-dependent increase in NET1 protein levels (Figure 3B). Treatment with $20 \mu \mathrm{M}$ LPA was chosen for subsequent analysis and it resulted in a 10-fold significant increase in NET1 mRNA expression $(P<0.05)$ (Figure 3A). Using a $12 \mathrm{~h}$ wound-healing scratch assay to monitor cell migration, treatment with LPA was shown to result in $78 \%$ wound healing $(P<0.005)$ in comparison with control cells in which $12 \%$ healing was achieved $(P<0.05)$ (Figure 3C and D). Lysophosphatidic acid-induced expression of NET1 was blocked by siRNA-mediated knockdown of NET1 (Figure 4A). Treatment with LPA was insufficient at restoring normal levels of NET1 in cells in which NET1 knockdown had been achieved. Inhibition of RhoA activation with C3 exoenzyme did not effect LPA-driven NET1 expression and furthermore C3 exoenzyme alone had no effect on NET1 expression (Figure 4A).

The above strategy was used to delineate the activation status of RhoA in AGS cells. Treatment of AGS cells with LPA resulted in a significant increase in NET1 mRNA expression $(P<0.05)$ (Figure 4A) with associated increase in the amount of active RhoA protein, while not affecting the level of total RhoA protein (Figure 4B). Lysophosphatidic acid was shown to increase the levels of active RhoA in AGS gastric cancer cells (Figure 4B). Further to our finding that NET1 knockdown resulted in a reduction in the level of active RhoA (Figure 1C), the activation of RhoA by LPA was also inhibited in cells in which RNAi-mediated knockdown of NET1 mRNA was achieved (Figure 4B). Although LPA in the presence of $\mathrm{C} 3$ exoenzyme resulted in increased NET1 expression, this same treatment resulted in an inhibition of RhoA activation (Figure 4B). Treatment of cells with C3 exoenzyme resulted in a complete inhibition of RhoA activation (Figure 4B).

The effects of altered NET1 and RhoA levels on tumour cell chemotaxis were examined in vitro. Using a $12 \mathrm{~h}$ wound-healing scratch assay to monitor cell migration, treatment with LPA was shown to result in $78 \%$ wound healing $(P<0.005)$ in comparison with control cells in which $12 \%$ healing was achieved $(P<0.05)$ (Figure $3 \mathrm{C}$ and D). Treatment with LPA induced a $70 \%$ increase in AGS gastric cancer cell migration and $100 \%$ increased invasion in vitro $(P<0.05)$ (Figure 4C and D). Knockdown of NET1 significantly reduced the level of LPA-mediated chemotaxis. Lysophosphatidic acid-induced migration was reduced by $88 \%$ and LPA-induced invasion was reduced by $93 \%$ in cells in which NET1 was silenced $(P<0.05)$ (Figure $4 \mathrm{C}$ and D). Although LPA was shown to drive the expression of NET1 in the presence of the RhoA inhibitor C3 exoenxyme (Figure 4A), these cells had 91 and $96 \%$ reduced migratory and invasive capabilities in comparison with cells treated with LPA alone $(P<0.05)$ (Figure $4 \mathrm{C}$ and $D)$. Treatment with C3 exoenzyme alone resulted in $90 \%$ reduced migration and $86 \%$ reduced invasion in comparison with control cells $(P<0.05)$ (Figure $4 \mathrm{C}$ and $\mathrm{D})$. 

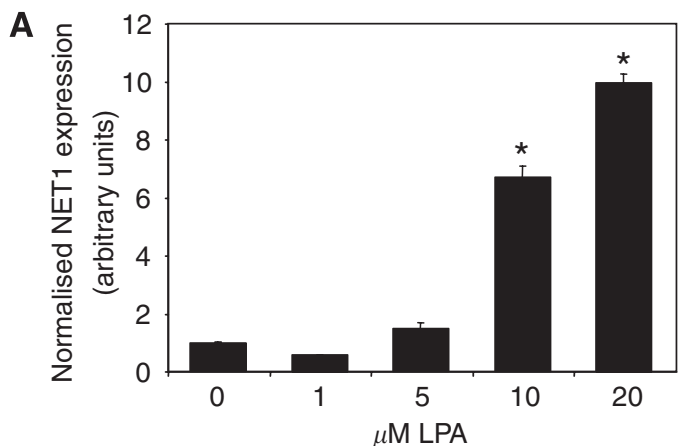

B
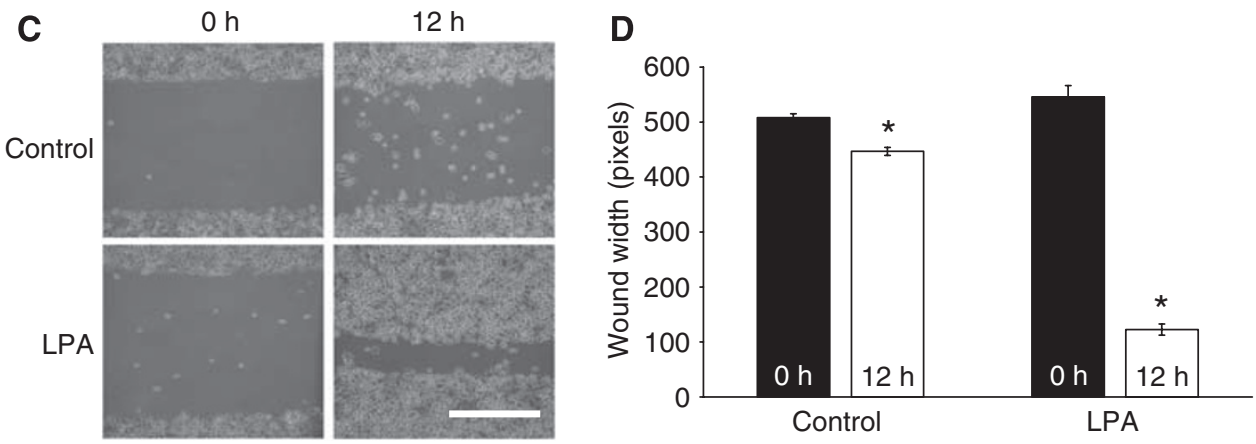

Figure 3 Lysophosphatidic acid (LPA) drives NETI expression in a dose-responsive manner and promotes AGS cell migration. (A) Expression of NET I mRNA in AGS gastric cancer cells treated with various doses of LPA for $4 \mathrm{~h}$, as detected by quantitative real-time PCR $(* P<0.05)$. (B) Expression of NETI protein in AGS gastric cancer cells treated with various doses of LPA for $4 \mathrm{~h}$, as detected by western immunoblot, respectively. (C) The effect of $20 \mu \mathrm{M}$ LPA on AGS cell migration using a wound-healing assay in comparison with control AGS cells. Cells were grown to confluence, and immediately following the 'wound' fresh media was added with or without LPA. Cell migration was measured I $2 \mathrm{~h}$ after the 'wound'. Bar, $200 \mu \mathrm{m}$. (D) Graphical representation of the effect of LPA on wound healing. (*P<0.05). Error bars represent standard deviation of triplicate experiments.

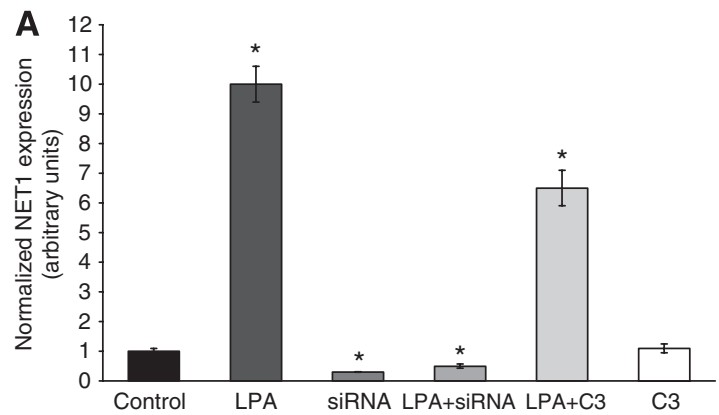

B
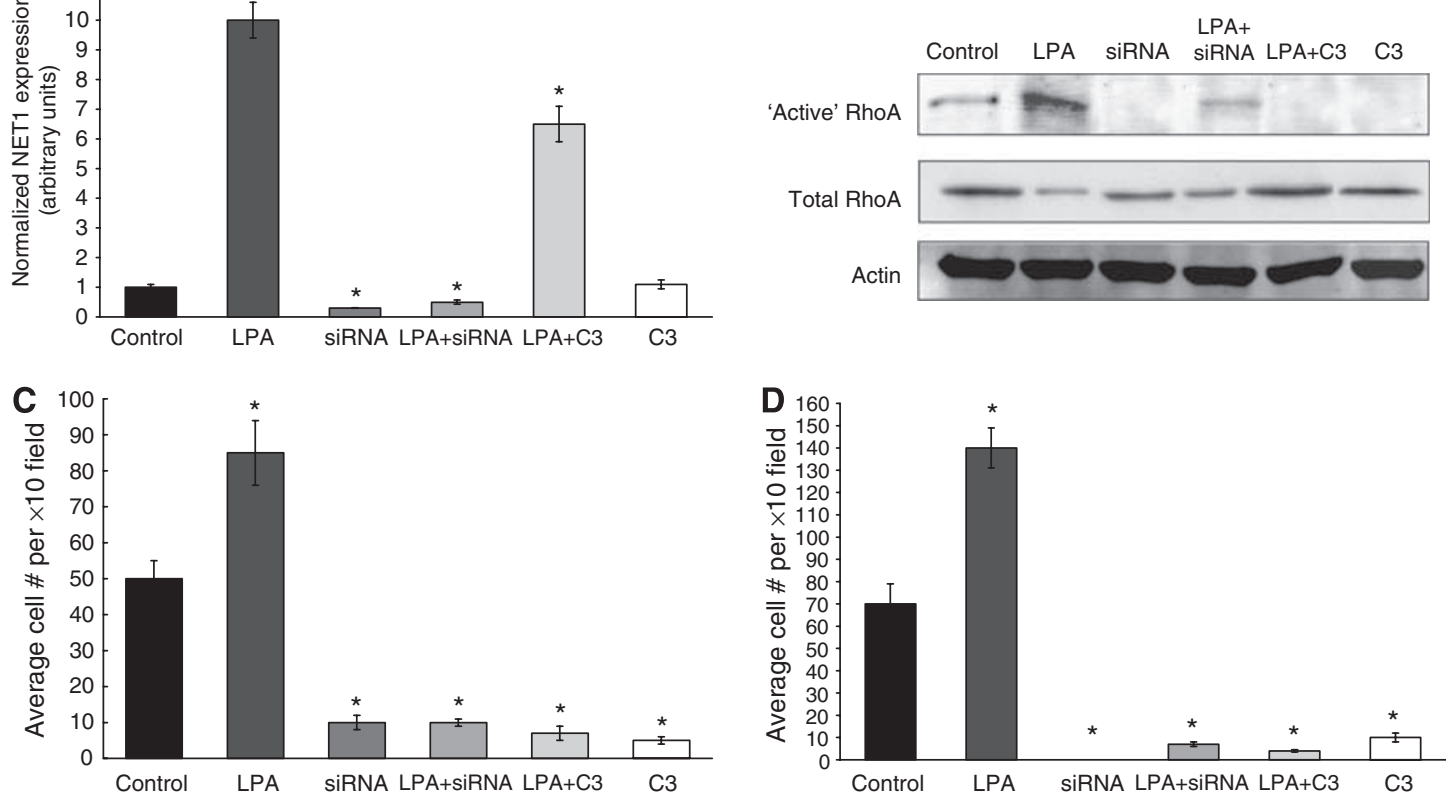

Figure 4 Lysophosphatidic acid (LPA)-induced NETI expression drives AGS cell invasion by RhoA activation. (A) Effect of LPA, NETI siRNA and C3 exoenzyme on NETI mRNA expression. Cells were treated with siRNA for $48 \mathrm{~h}$ before treatment with or without LPA or C3 exoenzyme, $4 \mathrm{~h}$ after which cells were lysed and analysed by real-time PCR. Error bars represent the standard deviation of triplicate experiments. (B) Western blot analysis showing the effect of LPA, NETI siRNA and C3 exoenzyme on levels of 'active' and total RhoA and $\beta$-actin. Cells were treated with siRNA for $48 \mathrm{~h}$ before treatment with or without LPA or C3, $4 \mathrm{~h}$ after which cells were lysed and analysed bya western immunoblot. All immunoblot analysis was repeated in triplicate. (C) The in vitro migration of AGS cells treated with LPA, NETI siRNA and C3 exoenzyme. Cells were treated with siRNA for $48 \mathrm{~h}$ before treatment with or without LPA or C3, 24h after which the effect on migration was assessed. Error bars represent the standard deviation of triplicate experiments. (D) The effect of LPA, NETI siRNA and C3 exoenzyme on the in vitro invasion of AGS cells. Cells were treated with siRNA for $48 \mathrm{~h}$ before treatment with or without LPA or C3, 24h after which the effect on invasion was assessed. Error bars represent the standard deviation of triplicate analysis (*P $<0.05$ ). 

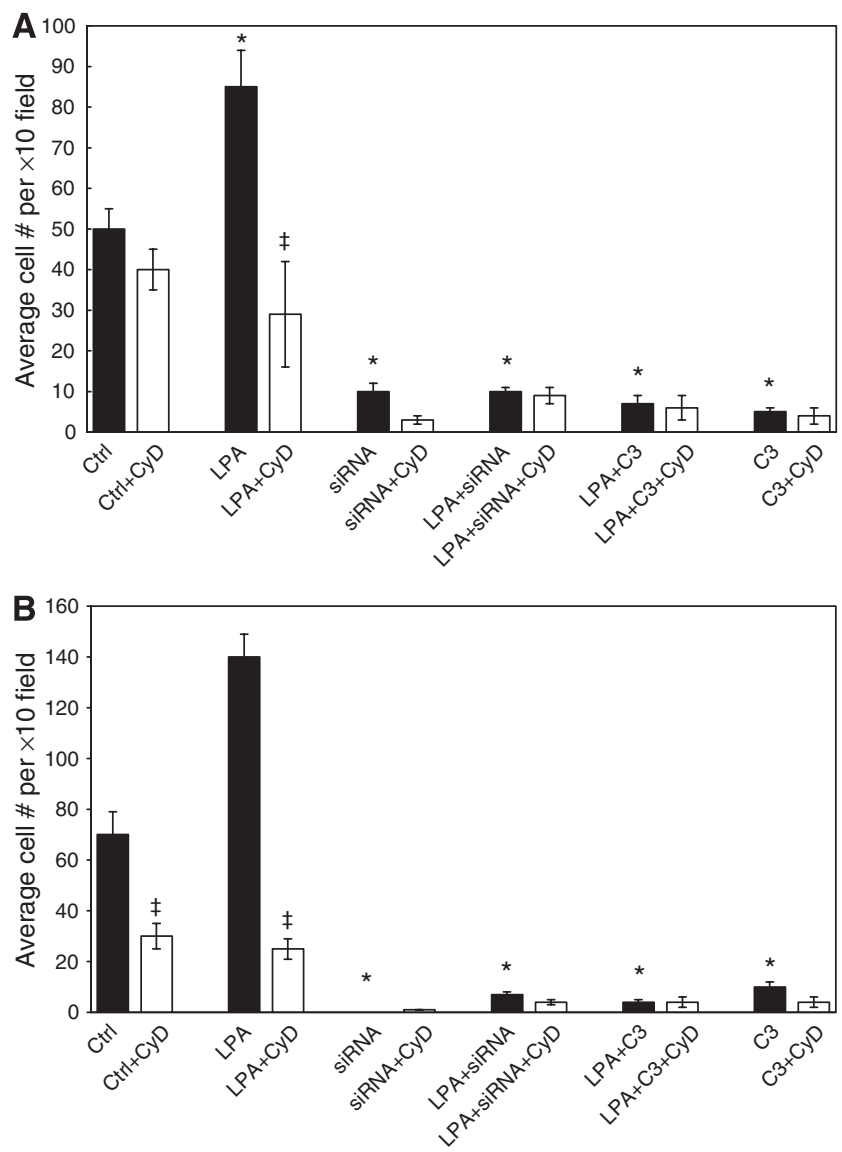

Figure 5 Lysophosphatidic acid (LPA)-mediated cell invasion occurs by cytoskeletal rearrangement events. The effect of cytochalasin D (CyD) on (A); the in vitro migration of AGS gastric cancer cells treated with LPA, NETI siRNA or C3 exoenzyme, and (B) the in vitro invasion of these cells. Cells were treated with siRNA for $48 \mathrm{~h}$ before treatment with or without LPA, C3 or CyD, 24h after which the effects on invasion and migration

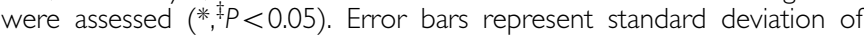
triplicate experiments.

\section{The role of cytoskeletal remodelling events in NET1-} mediated gastric cancer cell migration and invasion

Having demonstrated a role for NET1 in mediating invasion, the effect of NET1 on cytoskeletal rearrangements was investigated. Cytochalasin D, an inhibitor of actin filament rearrangement significantly inhibited LPA-induced AGS cell migration and invasion by 67 and $84 \%$, respectively $(P<0.05)$ (Figure $5 \mathrm{~A}$ and $B)$. The invasion of control cells was also significantly reduced by $46 \%$ in the presence of CyD $(P<0.05)$ (Figure $5 \mathrm{~B})$. Cytochalasin D reduced the migration of control cells, although this reduction was not statistically significant (Figure 5A). Control cells and cells treated with LPA displayed well-defined actin filament structures (Figure 6A and B). Knockdown of NET resulted in cell rounding and a loss of definition in the actin cytoskeleton (Figure 6C) in comparison with cells treated with nonspecific siRNA (Figure 5A). Cells treated with LPA in which NET1 knockdown had been achieved also appeared round with less actin cytoskeleton organisation than control cells (Figure 6D).

\section{DISCUSSION}

We have previously identified NET1 as being upregulated in GA and to participate in gastric cancer cell proliferation and invasion (Leyden et al, 2006). In this current study, our aim was to further characterise the mechanisms underpinning NET1-mediated gastric cancer cell invasion (Figure 7).

Neuroepithelial cell transforming gene 1 is a GEF and an activator of RhoA (Leyden et al, 2006). Rho proteins, once activated, stimulate signalling in multiple pathways by binding to downstream effector proteins, modulating their activities and thereby regulating a range of cellular processes. Rho-family proteins are regulators of the actin cytoskeleton, cell-cycle progression and gene transcription, and have been implicated in cellular processes such as adhesion and migration, cellular morphogenesis and polarisation (Hall, 1998; Evers et al, 2000; Sahai and Marshall, 2002; Raftopoulou and Hall, 2004).

Using an RNAi-based approach, NET1 was shown to activate RhoA with knockdown of NET1 inhibiting the activation of RhoA by $84 \%$ (Figure $1 \mathrm{C}$ and $\mathrm{D}$ ). A second siRNA set was used to confirm NET1-mediated activation of RhoA, thereby ruling out off target effects (Supplementary Figure 2). Interestingly, NET1 knockdown resulted in a decrease in total RhoA levels, thereby suggesting that as well as regulating RhoA activation, NET1 may play a role in RhoA transcription. This is the first report that NET1 drives the activation of RhoA in GA. This finding strengthens the role of RhoA in gastric cancer and furthermore elaborates on the biology of NET1, a protein whose role in the disease is not yet fully understood. Interestingly, NET1 knockdown did not result in a change in the levels of either RhoB or RhoC (Supplementary Figure 3), thereby supporting its role as a RhoAspecific exchange factor in AGS cells.

Because of the established role of RhoA in cell migration and invasion (Itoh et al, 1999; Somlyo et al, 2000; Ridley, 2001), the effect of NET1 knockdown and therefore reduced levels of active RhoA, on these cellular processes in AGS cells was investigated. As expected, knockdown of NET1 resulted in a significant decrease in gastric cancer cell migration, as assed using the in vitro woundhealing assay (Figure 2A and B). The importance of NET1 in tumour cell chemotaxis was further demonstrated using the transwell system, whereby AGS cells, in which NET1 knockdown has been achieved, were shown to be less migratory and invasive in vitro (Figure $2 \mathrm{C}$ and $\mathrm{D}$ ). These data highlight the importance of RhoA biology in the gastric cancer invasive programme as well as establishing the role of NET1 in this setting.

Rho activation is frequently mediated through various cellsurface receptors, including the tyrosine kinase, cytokine and adhesion receptors and also the G-protein-coupled receptors (Kjoller and Hall, 1999; Dorsam and Gutkind, 2007). Lysophosphatidic acid is a well-established driver of RhoA activation and has been shown to drive RhoA-mediated cytoskeletal rearrangement events (Hart et al, 1998; Mao et al, 1998; Mills and Moolenaar, 2003). Lysophosphatidic acid is known to promote the migration of colorectal cancer cells and to furthermore drive their secretion of proangiogenic factors such as VEGF, which is essential for metastasis (Shida et al, 2003). In this study, LPA was shown to drive the expression of NET1 mRNA and protein in a dose-dependent manner (Figure 3A and B). Although the role of LPA in transcription remains to be fully understood, reports suggest that LPA-induced gene expression is mediated through peroxisome proliferator-activated receptor, a transcriptional factor identified as an intracellular LPA receptor (McIntyre et al, 2003). Furthermore, we have previously shown that NET1 mRNA expression is also driven by treatment with $\mathrm{TNF} \alpha$ (Leyden et al, 2006). Although not explored in this study, the potential relationship between TNF $\alpha$ and LPA in driving NET1 mRNA transcription remains to be established. Indeed treatment with $\mathrm{TNF} \alpha$ has been shown to drive the expression of LPA receptor mRNA, which is one mechanism worth exploring in the future (Zhao et al, 2008). As expected, treatment with LPA resulted in an increase in the levels of active RhoA (Figure 4B). Cells treated with LPA were significantly more migratory and invasive than control cells (Figures 3C, D and 4C, D). Although LPA resulted in a 10-fold 

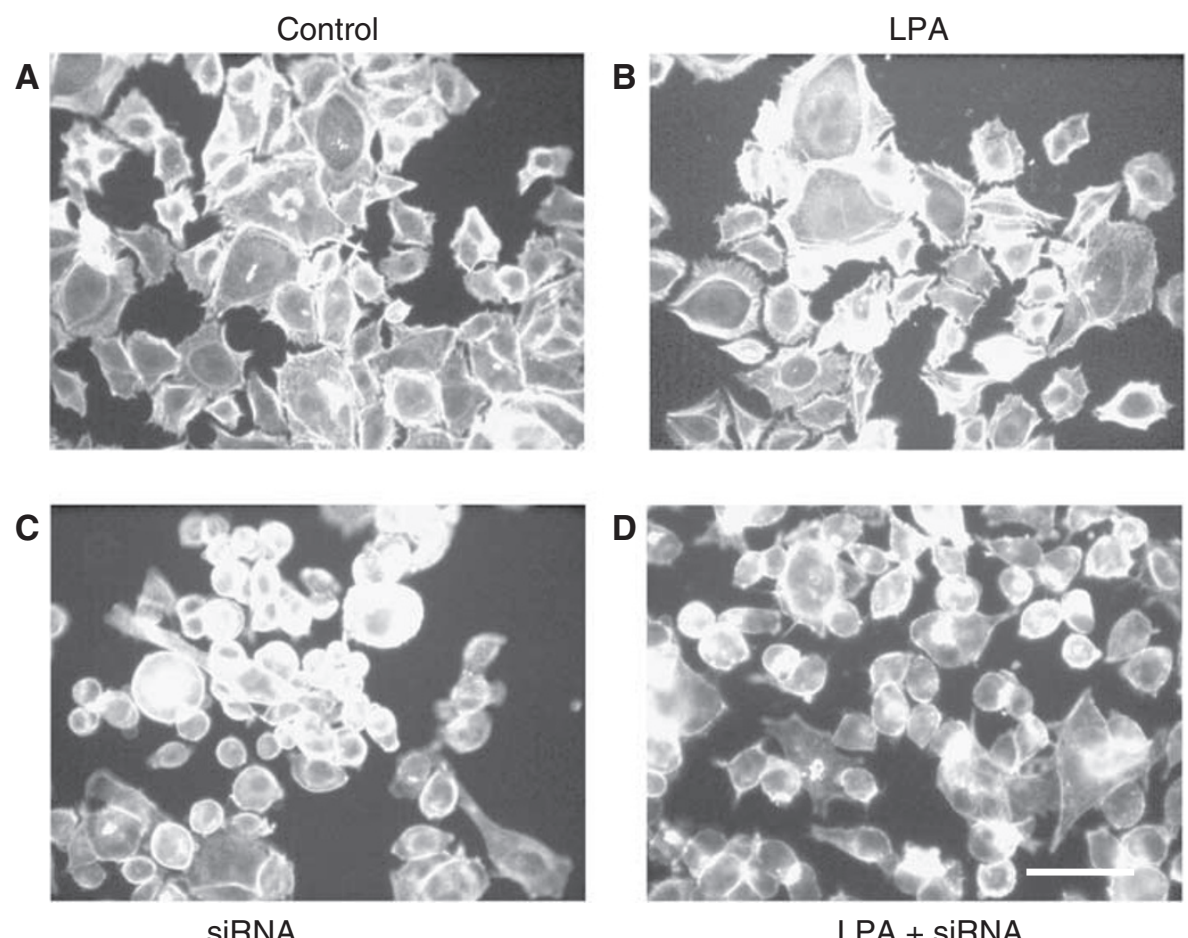

SiRNA

LPA + SiRNA

Figure 6 NETI knockdown blocks the organisation of the actin cytoskeleton. Fluorescence microscopy of F-actin formation in (A) control AGS cells, (B) cells treated with LPA, $(\mathbf{C})$ cells in which NETI knockdown was achieved and (D) cells treated with LPA and NETI siRNA. Cells were treated with siRNA for $48 \mathrm{~h}$ before treatment with or without LPA, $4 \mathrm{~h}$ after which the effect on actin polymerisation was assessed. F-actin was detected using TRITCconjugated phalloidin (green). Nuclei were counter stained using propidium iodide (orange). Bar $40 \mu \mathrm{m}$.

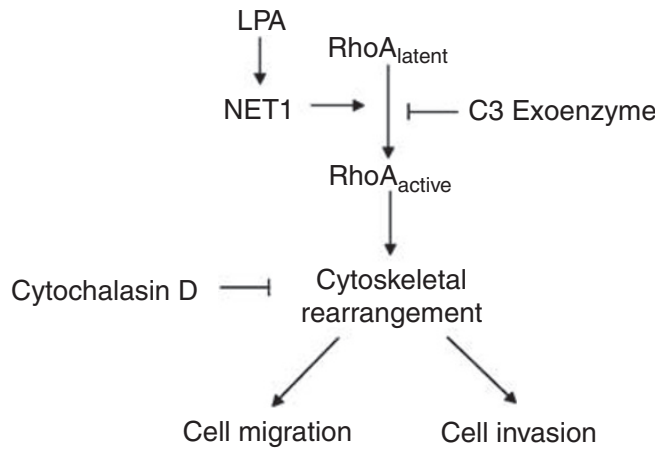

Figure 7 Summary of the mechanism of NETI-mediated tumour migration and invasion. In this study lysophosphatidic acid (LPA) was shown to drive the expression of NETI, which increased the activation of RhoA (a process that was inhibited by $\mathrm{C} 3$ exoenzyme). Knockdown of NETI was shown to reduce the levels of active RhoA and also a loss in the organisation of the cytoskeletal architecture. Knockdown of NETI furthermore resulted in a decrease in the levels of tumuor cell migration and invasion.

increase in NET1 mRNA levels, this did not translate into a 10-fold increase in migration and invasion, suggesting that NET1 protein levels may be at a plateau in these cells. Knockdown of NET1 was shown to inhibit LPA-induced activation of RhoA (Figure 4B) and furthermore LPA-induced cell migration and invasion (Figure $4 \mathrm{C}$ and D). Interestingly, the level of active RhoA in these cells was similar to control cells, yet their invasion and migration was reduced, demonstrating that NET1 is not essential for LPAmediated activation of RhoA, as well as suggesting that NET1 may mediate migration and invasion through other mechanisms besides RhoA. Inhibition of RhoA activation with $\mathrm{C} 3$ exoenzyme did not affect LPA-driven NET1 expression and furthermore C3 exoenzyme alone had no effect on NET1 expression (Figure 4A). Taken together, these data show that NET1 expression and activity lie upstream of RhoA biology and that LPA is driving RhoA activation through NET1. Although LPA was shown to increase the expression of NET1 in the presence of C3 exoenzyme (Figure 4A), these cells had reduced levels of migration and invasion (Figure $4 \mathrm{C}$ and D). As well as highlighting the importance of NET1 and RhoA in cell invasion, these data demonstrate that both NET1 and RhoA bioactivities are essential in LPA-induced gastric cancer cell migration and invasion. Furthermore, we have shown that NET1 inhibition is as potent at curbing cell migration and invasion as a RhoA inhibitor. These data firmly establish the role of enhanced NET1 expression in this disease, whereby NET1 is of key importance in gastric cancer cell invasion.

Further to our data demonstrating that LPA drives tumour cell invasion through both NET1 and RhoA, we next investigated the role of the cytoskeleton in these processes. Regulation of the cytoskeleton is a major function of RhoA activity (Hall, 1998; Matsui et al, 1998). Cytochalasin D was used in these studies. Cytochalasin D is a mycotoxin that inhibits both the association and dissociation of actin filament subunits by causing disruption of actin filaments and furthermore the inhibition of actin polymerisation (Goddette and Frieden, 1986). Treatment with CyD caused reduced migration and invasion of AGS cancer cells (Figure 5A and B). Lysophosphatidic acid-induced cell migration and invasion were dramatically reduced with $C y D$ treatment (Figure 5A and B). These data support our hypothesis that LPAinduced chemotaxis of AGS gastric cancer cells occurs by cytoskeletal rearrangement events, which are mediated at least in part by NET1.

We have shown that LPA-mediated cell invasion involves increased NET1 expression and RhoA activation. To further elucidate the underlying mechanism promoting cell invasion, the effect of various treatments on the actin cytoskeleton were 
investigated as Rho proteins are known key mediators of the actin organisation (Van Aelst and D'Souza-Schorey, 1997; Ellis and Mellor, 2000). Control AGS cells demonstrated defined actin filament organisation (Figure 6A). Upon treatment with LPA, these filaments became more pronounced and cells appeared elongated and stretched (Figure 6B). Lysophosphatidic acid is known to mediate actin cytoskeletal changes by Rho proteins and furthermore to facilitate cell migration (Jalink et al, 1994; Van Leeuwen et al, 2003). Cells in which NET1 knockdown had been achieved failed to demonstrate the organisation of actin filaments observed in control or LPA-treated cells (Figure 6C). These cells have been shown to be less migratory and invasive than control cells (Figure $4 \mathrm{C}$ and D), suggesting that NET1 mediates gastric cancer cell invasion through cytoskeletal-dependent events.

This study further elaborates on the role of NET1, a novel GA-associated GEF in the disease. We have previously shown that NET1 is enhanced in gastric cancer tissue in comparison with normal tissue (Leyden et al, 2006) and this study further elucidates the mechanism by which NET1 mediates the progression of the disease. We have shown that NET1 is a key player in LPA-induced activation of RhoA and the subsequent migration and invasion of

\section{REFERENCES}

Advani AS, Pendergast AM (2002) Bcr-Abl variants: biological and clinical aspects. Leuk Res 26: 713-720

Bishop AL, Hall A (2000) Rho GTPases and their effector proteins. Biochem J 348(Part 2): $241-255$

Chan AM, Takai S, Yamada K, Miki T (1996) Isolation of a novel oncogene, NET1, from neuroepithelioma cells by expression cDNA cloning. Oncogene 12: 1259-1266

Chiou CC, Chan CC, Sheu DL, Chen KT, Li YS, Chan EC (2001) Helicobacter pylori infection induced alteration of gene expression in human gastric cells. Gut 48: 598-604

de Manzoni G, Pedrazzani C, Di Leo A, Bonfiglio M, Tasselli S, Guglielmi A, Cordiano C (2001) Metastases to the para-aortic lymph nodes in adenocarcinoma of the cardia. Eur J Surg 167: 413-418

Dorsam RT, Gutkind JS (2007) G-protein-coupled receptors and cancer. Nat Rev Cancer 7: 79-94

Ellis S, Mellor H (2000) The novel Rho-family GTPase rif regulates coordinated actin-based membrane rearrangements. Curr Biol 10: 1387-1390

Evers EE, Zondag GC, Malliri A, Price LS, ten Klooster JP, van der Kammen RA, Collard JG (2000) Rho family proteins in cell adhesion and cell migration. Eur J Cancer 36: 1269-1274

Goddette DW, Frieden C (1986) Actin polymerization. The mechanism of action of cytochalasin D. J Biol Chem 261: 15974-15980

Hall A (1998) Rho GTPases and the actin cytoskeleton. Science 279: 509-514

Hart MJ, Jiang X, Kozasa T, Roscoe W, Singer WD, Gilman AG, Sternweis PC, Bollag G (1998) Direct stimulation of the guanine nucleotide exchange activity of p115 RhoGEF by Galpha13. Science 280: 2112-2114 Hohenberger P, Gretschel S (2003) Gastric cancer. Lancet 362: 305-315

Itoh K, Yoshioka K, Akedo H, Uehata M, Ishizaki T, Narumiya S (1999) An essential part for Rho-associated kinase in the transcellular invasion of tumor cells. Nat Med 5: 221-225

Jalink K, van Corven EJ, Hengeveld T, Morii N, Narumiya S, Moolenaar WH (1994) Inhibition of lysophosphatidate- and thrombin-induced neurite retraction and neuronal cell rounding by ADP ribosylation of the small GTP-binding protein Rho. J Cell Biol 126: 801-810

Kawasaki Y, Sato R, Akiyama T (2003) Mutated APC and Asef are involved in the migration of colorectal tumour cells. Nat Cell Biol 5: 211-215

Kjoller L, Hall A (1999) Signaling to Rho GTPases. Exp Cell Res 253: 166-179

Leyden J, Murray D, Moss A, Arumuguma M, Doyle E, McEntee G, O'Keane C, Doran P, MacMathuna P (2006) Net1 and Myeov: computationally identified mediators of gastric cancer. Br J Cancer 94: 1204-1212

Mao J, Yuan H, Xie W, Wu D (1998) Guanine nucleotide exchange factor GEF115 specifically mediates activation of Rho and serum response factor by the G protein alpha subunit Galpha13. Proc Natl Acad Sci USA 95: $12973-12976$

Matsui T, Maeda M, Doi Y, Yonemura S, Amano M, Kaibuchi K, Tsukita S, Tsukita S (1998) Rho-kinase phosphorylates $\mathrm{COOH}$-terminal threonines of ezrin/radixin/moesin (ERM) proteins and regulates their head-to-tail association. J Cell Biol 140: 647-657 gastric tumour cells. NET1 inhibition was as effective at reducing cell invasion as treatment with the RhoA inhibitor, C3 exoenzyme or the inhibitor of cytoskeletal rearrangement $\mathrm{CyD}$, highlighting its importance in this setting. As NET1 is important to the invasive phonotype, we therefore propose that NET1 is an ideal potential therapeutic target in this disease.

\section{ACKNOWLEDGEMENTS}

This study was supported by the Irish Cancer Society, and the Irish Government's Programme for Research in Third Level Institutions, and the Mater College for Postgraduate Education and Research. Thanks for technical assistance to $\mathrm{Dr}$ Alfonso Blanco Fernández, Flow Cytometry Core Facility, UCD Conway Institute, Dublin 4, Ireland.

Supplementary Information accompanies the paper on British Journal of Cancer website (http://www.nature.com/bjc)

McIntyre TM, Pontsler AV, Silva AR, St Hilaire A, Xu Y, Hinshaw JC, Zimmerman GA, Hama K, Aoki J, Arai H, Prestwich GD (2003) Identification of an intracellular receptor for lysophosphatidic acid (LPA): LPA is a transcellular PPARgamma agonist. Proc Natl Acad Sci USA 100: $131-136$

Mills GB, Moolenaar WH (2003) The emerging role of lysophosphatidic acid in cancer. Nat Rev Cancer 3: 582-591

Mizuarai S, Yamanaka K, Kotani H (2006) Mutant p53 induces the GEF-H1 oncogene, a guanine nucleotide exchange factor-H1 for RhoA, resulting in accelerated cell proliferation in tumor cells. Cancer Res 66: 6319-6326

Nathke I (2006) Cytoskeleton out of the cupboard: colon cancer and cytoskeletal changes induced by loss of APC. Nat Rev Cancer 6: $967-974$

Poste G, Fidler IJ (1980) The pathogenesis of cancer metastasis. Nature 283: $139-146$

Raftopoulou M, Hall A (2004) Cell migration: Rho GTPases lead the way. Dev Biol 265: 23 - 32

Ridley AJ (2001) Rho GTPases and cell migration. J Cell Sci 114: 2713-2722 Rossman KL, Der CJ, Sondek J (2005) GEF means go: turning on RHO GTPases with guanine nucleotide-exchange factors. Nat Rev Mol Cell Biol 6: $167-180$

Sahai E, Marshall CJ (2002) RHO-GTPases and cancer. Nat Rev Cancer 2: $133-142$

Sander EE, van Delft S, ten Klooster JP, Reid T, van der Kammen RA, Michiels F, Collard JG (1998) Matrix-dependent Tiam1/Rac signaling in epithelial cells promotes either cell-cell adhesion or cell migration and is regulated by phosphatidylinositol 3-kinase. J Cell Biol 143: 1385-1398

Shida D, Kitayama J, Yamaguchi H, Okaji Y, Tsuno NH, Watanabe T, Takuwa Y, Nagawa H (2003) Lysophosphatidic acid (LPA) enhances the metastatic potential of human colon carcinoma DLD1 cells through LPA1. Cancer Res 63: 1706-1711

Somlyo AV, Bradshaw D, Ramos S, Murphy C, Myers CE, Somlyo AP (2000) Rho-kinase inhibitor retards migration and in vivo dissemination of human prostate cancer cells. Biochem Biophys Res Commun 269: 652 - 659 Symons M, Rusk N (2003) Control of vesicular trafficking by Rho GTPases. Curr Biol 13: R409-R418

Van Aelst L, D’Souza-Schorey C (1997) Rho GTPases and signaling networks. Genes Dev 11: 2295-2322

Van Leeuwen FN, Olivo C, Grivell S, Giepmans BN, Collard JG, Moolenaar WH (2003) Rac activation by lysophosphatidic acid LPA1 receptors through the guanine nucleotide exchange factor Tiam1. J Biol Chem 278: $400-406$

Wang W, Goswami S, Sahai E, Wyckoff JB, Segall JE, Condeelis JS (2005) Tumor cells caught in the act of invading: their strategy for enhanced cell motility. Trends Cell Biol 15: 138-145

Zhao C, Fernandes MJ, Prestwich GD, Turgeon M, Di Battista J, Clair T, Poubelle PE, Bourgoin SG (2008) Regulation of lysophosphatidic acid receptor expression and function in human synoviocytes: implications for rheumatoid arthritis? Mol Pharmacol 73: 587-600 